\title{
IL-33 reflects dynamics of disease activity in patients with autoimmune hemolytic anemia by regulating autoantibody production
}

\author{
Xiangmao Bu ${ }^{1}$, Tenglong Zhang ${ }^{2}$, Chunhong Wang ${ }^{3}$, Tao Ren ${ }^{3 *}$ and Zhenke Wen ${ }^{4,5^{*}}$
}

\begin{abstract}
Background: Autoimmune hemolytic anemia (AlHA), a life-threatening anemia with rapid onset, is caused by autoantibody directed to self red blood cells (RBCs). Currently, mechanisms underlying AlHA pathogenesis are largely undefined. Here we explored the correlation of IL-33 with AlHA disease activity and evaluated IL-33 based therapeutics in AlHA treatment.

Methods: Thirty patients diagnosed with AlHA of warm-type autoantibodies without treatment were enrolled and followed up for 6 months. Levels of cytokines including IL-33, IL-4, IL-6 and IL-13 was determined with ELISA. AIHA disease activity was presented by levels of reticulocyte count, hemoglobin and lactate dehydrogenase. Serum RBCbound IgG autoantibody was detected using anti-lgG antibody with flow cytometry. To evaluate the effect of IL-33 blockade on AlHA development, groups of B6 mice were immunized with rat RBCs plus recombinant IL-33 protein or IL-33 neutralizing antibody respectively and detected for levels of anti-RBC antibody, frequency of reticulocytes and destruction of transfused syngeneic mouse RBCs.
\end{abstract}

Results: Serum level of IL-33 was higher in AlHA patients compared with healthy individuals. Of interest, serum IL-33 was positively correlated with AlHA disease activity and sensitive to their changes in AlHA patients under clinical management. Mechanistically, IL-33 could promote the production of anti-RBC autoantibody. Serum IL-33 was closely associated with serum anti-RBC autoantibody and sensitive to their changes in AlHA patients. Accordingly, blockade of IL-33 interfered with AlHA incidence and ameliorated disease activity. Vice vasa, enforced IL-33 promoted AlHA incidence and disease activity.

Conclusions: IL-33 was a potential biomarker for monitoring disease activity and therapeutic response in AlHA patients. Targeting IL-33 was a promising strategy for controlling autoantibody production in AlHA patients.

Keywords: Autoimmune hemolytic anemia, IL-33, Red blood cell, Anti-RBC autoantibody

\section{Background}

Autoimmune hemolytic anemia (AIHA), a life-threatening anemia with rapid onset, is defined by enhanced destruction of red blood cells (RBCs) because of the production and accumulation of anti-RBC autoantibodies

\footnotetext{
*Correspondence: rentaosh@126.com; wenzk@126.com

${ }^{3}$ Department of Respiratory Medicine, East Hospital, Tongji University

School of Medicine, 150 Jimo Road, Shanghai 200120, China

${ }_{5}$ Present Address: Division of Immunology and Rheumatology,

Stanford University School of Medicine, 269 Campus Drive West,

Stanford, CA 94305, USA

Full list of author information is available at the end of the article
}

$[1,2]$. AIHA can be classified by optimal temperature at which the anti-RBC autoantibodies bind to a patient's RBCs, and warm AIHA accounts for about $80 \%$ of all AIHA cases [3]. In patients with warm AIHA, the antiRBC autoantibodies are usually IgG type and bind optimally to RBCs at $37^{\circ} \mathrm{C}$, causing $\mathrm{RBC}$ destruction by tissue macrophages $[1,4-6]$. Currently, therapeutic options for patients with AIHA are still limited due to the obscure pathogenesis.

Interleukin 33 (IL-33), a novel identified member of IL-1 family, can be produced in various tissues and cells, and orchestrate complex innate and adaptive immune 
responses [7-9]. Through binding to the orphan receptor ST2, IL-33 can induce gene expression of Th2-associated cytokines, promote Th2 type immune response, and thus play important roles in autoimmune diseases [9-11]. In rheumatoid arthritis, a higher level of IL-33 was detected in synovial fluid, serum, and was correlated with disease activity compared to moderate or low activity group or healthy controls $[12,13]$. Blockade of IL-33/ST2 interaction resulted in dramatically attenuated disease severity of rheumatoid arthritis [14]. In systemic lupus erythematosus, elevated serum IL-33 was reported in clinical patients and correlated with their disease activity [1517]. Disrupting IL-33 pathway resulted in reduced serum anti-dsDNA levels, alleviated proteinuria, and attenuated lupus nephritis in lupus-prone mice [18]. These findings demonstrated a possible role of IL-33 in production of IgG autoantibodies and development of autoimmune diseases. However, whether IL-33 functions in AIHA pathogenesis still remains unclear.

In this study, we investigated the potential role of IL-33 in AIHA pathogenesis in patients with warm AIHA. Our findings revealed an elevated level of serum IL-33 in patients with AIHA. Of interest, serum IL-33 was correlated with disease activity and sensitive to their changes in AIHA patients. IL-33 could facilitate production of IgG anti-RBC autoantibody from peripheral blood mononuclear cells in AIHA patients. Blockade of IL-33 restrained AIHA incidence and disease activity, while enforced IL-33 promoted the development of AIHA. These findings assigned IL-33 an important function involved in AIHA pathogenesis and provided clues for exploring new AIHA therapeutics.

\section{Methods}

\section{Patients}

Thirty patients diagnosed with AIHA of warm-type autoantibodies without treatment were enrolled and followed up for 6 months in this study. AIHA diagnosis was made with clinical and laboratory hemolytic signs plus positive direct anti-globulin test (DAT) as previously described [1]. Eighteen age- and sex-matched healthy controls were studied. Patients were considered as having active disease when an anemia and at least one of the following were present: reticulocyte count $>2 \%$, haptoglobin concentration $<500 \mathrm{mg} / \mathrm{L}$ and lactate dehydrogenase $(\mathrm{LDH})$ activity $>480 \mathrm{U} / \mathrm{L}$. All blood samples and clinical parameters were collected after an informed consent. This study was approved by the Ethics Committee of Tongji University and performed in accordance with the ethical standards laid down in the 1964 Declaration of Helsinki and its later amendments.

\section{Cell culture and reagents}

Peripheral blood mononuclear cells (PBMCs) were isolated from heparinized peripheral blood using FicollPaque density gradient (GE Healthcare). PBMCs were washed with phosphate-buffered saline (PBS) and cultured in RPMI 1640 medium with $10 \%$ FCS and $1 \%$ glutamine/penicillin/streptomycin (Life Technologies). Recombinant human IL-33 protein was purchased from R\&D Systems. Anti-human IgM antibody was purchased from eBioscience. Recombinant human CD40 Ligand (CD40L) was purchased from Life Technologies. For in vitro induction of anti-RBC IgG antibody, PBMCs $\left(1 \times 10^{6} / \mathrm{ml}\right)$ were cultured with anti-IgM $(10 \mu \mathrm{g} / \mathrm{ml})$ and CD40L $(10 \mathrm{ng} / \mathrm{ml})$ in the presence or absence of IL-33 (0-20 ng/ml). Six days later, the supernatants were collected and assayed for IgG antibodies with Human IgG total ELISA kit (eBioscience).

\section{Cytokines}

Serum level of IL-33 was determined with Human IL-33 Quantikine ELISA Kit (R\&D Systems). IL-4, IL-6 and IL-13 levels in culture supernatants were also detected with Human IL-4, IL-6 and IL-13 Quantikine ELISA Kits (R\&D Systems) respectively according to the manual's instructions.

\section{Anti-RBC IgG antibody}

Serum RBC-bound IgG autoantibody was detected with flow cytometry as described previously [1, 19]. Briefly, RBCs were freshly isolated, washed three times with warm PBS and then incubated with FITC anti-IgG antibody (Biolegend) at $37{ }^{\circ} \mathrm{C}$. The cells were immediately analyzed for percentage of RBC-bound antibody on a FACSCalibur flow cytometer (BD Biosciences). Collected data were analyzed with FlowJo software (TreeStar).

\section{Induction of murine AlHA}

Murine experiments were approved by the Ethics Committee of Tongji University. Female B6 mice between 8 and 10 weeks old were purchased from Shanghai Laboratory Animal Center and housed in specific pathogen free conditions. As previously described [1], induction of AIHA murine model was achieved by immunizing B6 mice with $2 \times 10^{8}$ rat RBCs on a weekly basis for 10 weeks. For transfusion study, mouse RBCs obtained from naive female B6 mice were labeled with PKH-26 (Sigma) and injected into control mice or those that had developed AIHA through tail-vein. Destruction of these syngeneic RBCs was represented by the clearance of fluorescent RBCs measured by flow cytometry. To show the clearance kinetics, injected RBCs at 1 min after injection were taken as $100 \%$, and the remaining $\mathrm{RBCs}$ were calculated at different time points as the average for each group of mice. 
For regulation of IL-33, B6 mice were injected with IL-33 neutralizing antibody or recombinant IL-33 respectively with isotype controls (R\&D Systems). Briefly, 1 day before rat RBCs immunization, mice were intraperitoneally injected with $100 \mu \mathrm{g}$ neutralizing antibodies per mouse or $2 \mu \mathrm{g}$ IL-33 per mouse respectively. Injections were repeated every 3 days during mice immunization.

\section{Statistical analyses}

Quantitative data were expressed as the mean \pm SD. Unpaired $t$ test and Pearson correlation were used for statistical analyses with PRISM 6.0 (GraphPad Software Inc.). A value of $\mathrm{P}<0.05$ was considered statistically significant.

\section{Results}

Serum level of IL-33 was elevated and correlated with disease activity in AIHA patients

We performed ELISA to determine serum levels of IL-33 in patients with AIHA and healthy controls, and results showed a significant higher level of serum IL-33 in AIHA patients (Fig. 1a). Further analysis of serum IL-33 in patients with active AIHA or with AIHA remission revealed a relative higher serum IL-33 in patients with active AIHA (Fig. 1b). These data indicated an involvement of IL-33 in AIHA pathogenesis. Thus, we detected the correlation between serum IL-33 and disease activity of active AIHA patients that is presented by the levels of reticulocyte count, hemoglobin and lactate dehydrogenase (LDH). We found that serum level of IL-33 was closely correlated with the level of reticulocyte frequency, negatively correlated with the level of hemoglobin and positively correlated with the level of LDH activity in AIHA patients (Fig. 1c-e). These results demonstrated that serum IL-33 was closely correlated with disease activity of AIHA.

\section{Serum IL-33 was sensitive to changes of AIHA disease activity}

To further explore the correlation of serum IL-33 with AIHA disease activity, patients with active AIHA were followed up for 6 months and analyzed for their changes of serum IL-33 and disease activity. Results showed that changes of serum IL-33 were associated with the changes of hemoglobin, positively correlated with changes of reticulocyte frequency and LDH in AIHA patients (Fig. 2a-c). These findings suggested that serum IL-33 was sensitive to changes of AIHA disease activity.

\section{Serum IL-33 was associated with anti-RBC autoantibody production}

To investigate the possible mechanisms underlying the close relationship between serum IL-33 and disease activity of AIHA patients, we analyzed the correlation of serum IL-33 with anti-RBC autoantibody, which plays a central role in AIHA pathogenesis. We found that serum IL-33 was positively correlated with the antiRBC autoantibody level in AIHA patients (Fig. 3a). Further, changes of serum IL-33 was closely associated with changes of anti-RBC autoantibody (Fig. 3b). These results could partly explain the close correlation of serum IL-33 with AIHA disease activity and indicated an involvement of IL-33 in autoantibody production in AIHA patients.

\section{IL-33 contributed to anti-RBC autoantibody production}

To further elucidate why serum IL-33 was correlated with anti-RBC autoantibody level, we detected the effect of IL-33 on IgG anti-RBC antibody production in AIHA patients. When PBMCs isolated from patients with active AIHA were stimulated with anti-IgM plus CD40L in the presence of an increasing dose of recombinant human IL-33 protein, we found that IL-33 could promote the production of IgG anti-RBC antibody in a dose dependent manner (Fig. 4a). Vice vasa, blockade of IL-33 efficiently reduced the enhanced production of IgG anti-RBC antibody (Fig. 4b). Given the important roles of IL-4, IL-6 and IL-13 in antibody production, we further detect the production of these cytokines in response to IL-33 stimulation. We found that stimulation of PBMCs isolated from active AIHA patients with IL-33 protein resulted in significant higher production of IL-4, IL-6 and IL-13 (Fig. 4c-e). These findings demonstrated that IL-33 could increase Th2 cytokines release and contribute to autoantibody production in patients with AIHA.

\section{Blockade of IL-33 interfered with incidence and disease severity of AIHA}

To directly evaluate the potential role of IL-33 in AIHA pathogenesis, murine AIHA was induced by immunizing B6 mice with rat RBCs with or without IL-33 neutralizing antibody. Within 30 mice immunized with rat RBCs, 13 mice co-injected with isotype control IgG exerted high levels of anti-RBC antibody and reticulocytes (Additional file 1: Fig. S1, 5A, B). Consistent with this observation, these mice showed increased destruction of transfused syngeneic mouse RBCs (Fig. 5c). When B6 mice were coinjected with IL-33 neutralizing antibody, only 5 out of 30 mice developed AIHA as evidenced by the circulating levels of anti-RBC antibody and reticulocytes (Fig. 5a, b). Of note, the level of anti-RBC antibody was significantly decreased by co-injection with IL-33 neutralizing antibody (Fig. 5d). Thus, the destruction of transferred syngeneic mouse RBCs was inhibited in IL-33 neutralizing antibody co-immunized mice (Fig. 5c). To further confirm the effect of IL-33 on AIHA pathogenesis, groups of $\mathrm{B} 6$ mice were immunized with rat RBCs plus recombinant IL-33 protein. Results showed that enforced IL-33 significantly enhanced the incidence of AIHA 

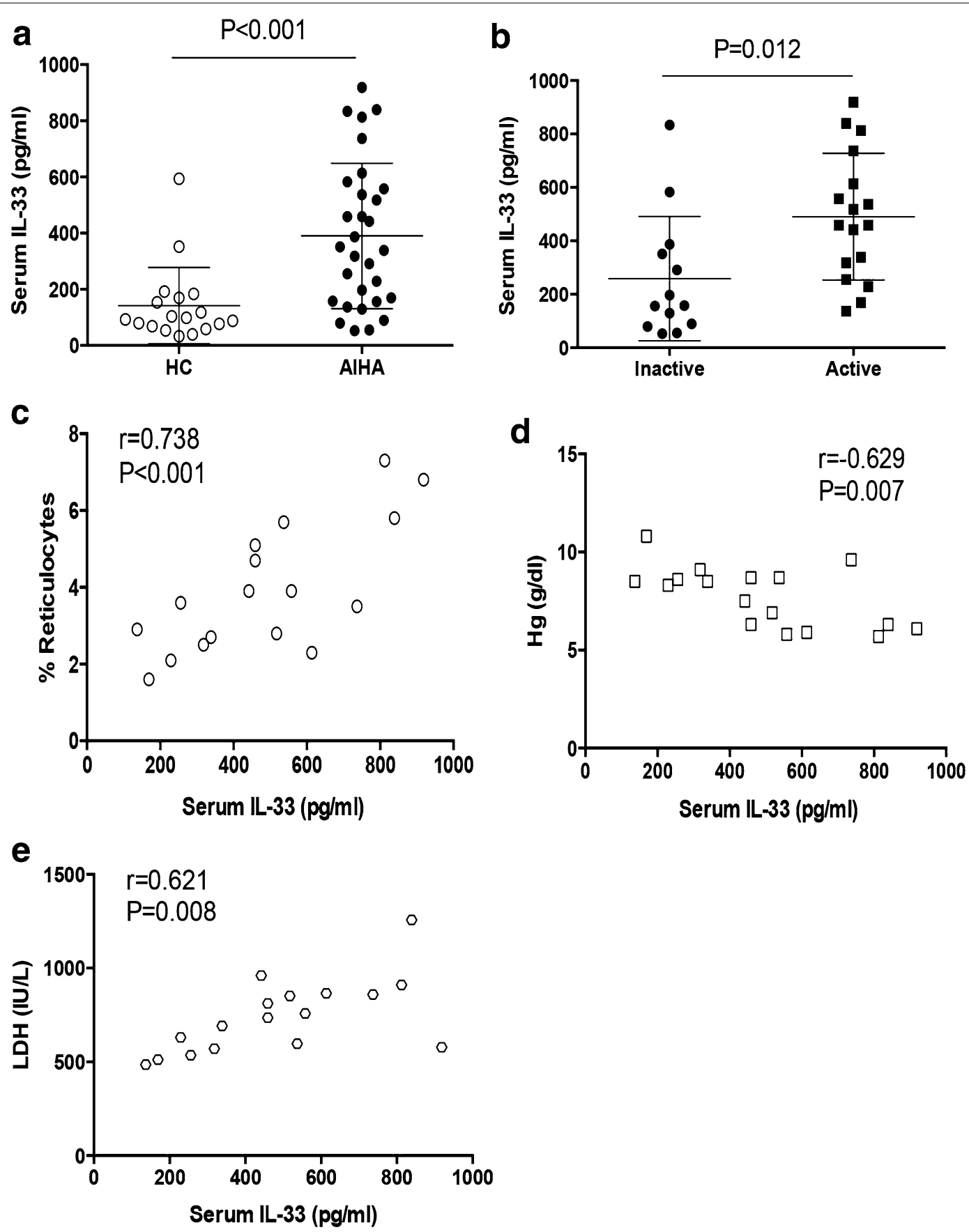

Fig. 1 Elevated serum IL-33 in AlHA patients was correlated with AlHA disease activity. a Serum IL-33 was determined in AlHA patients ( $\mathrm{n}=30$ ) and healthy controls $(n=18)$; b serum IL-33 was detected in patients with active AlHA $(n=17)$ or with inactive AlHA ( $n=13)$; c-e the correlation between serum IL-33 and levels of reticulocyte count (c), hemoglobin $(\mathbf{d})$ and lactate dehydrogenase (LDH, e) was analyzed in active AlHA patients $(n=17)$. One dot represented the result from one patient

and elevated the generation of $\operatorname{IgG}$ anti-RBC antibody (Fig. 5e-h). These results regarded IL-33 as an important regulator in AIHA pathogenesis and suggested that blockade of IL-33 was a promising strategy to control AIHA disease.

\section{Discussion}

AIHA is an autoimmune disorder caused by production of autoantibody that directed against self red blood cells $[20,21]$. Therefore, intervention of anti-RBC autoantibody production is essential for AIHA treatment. 

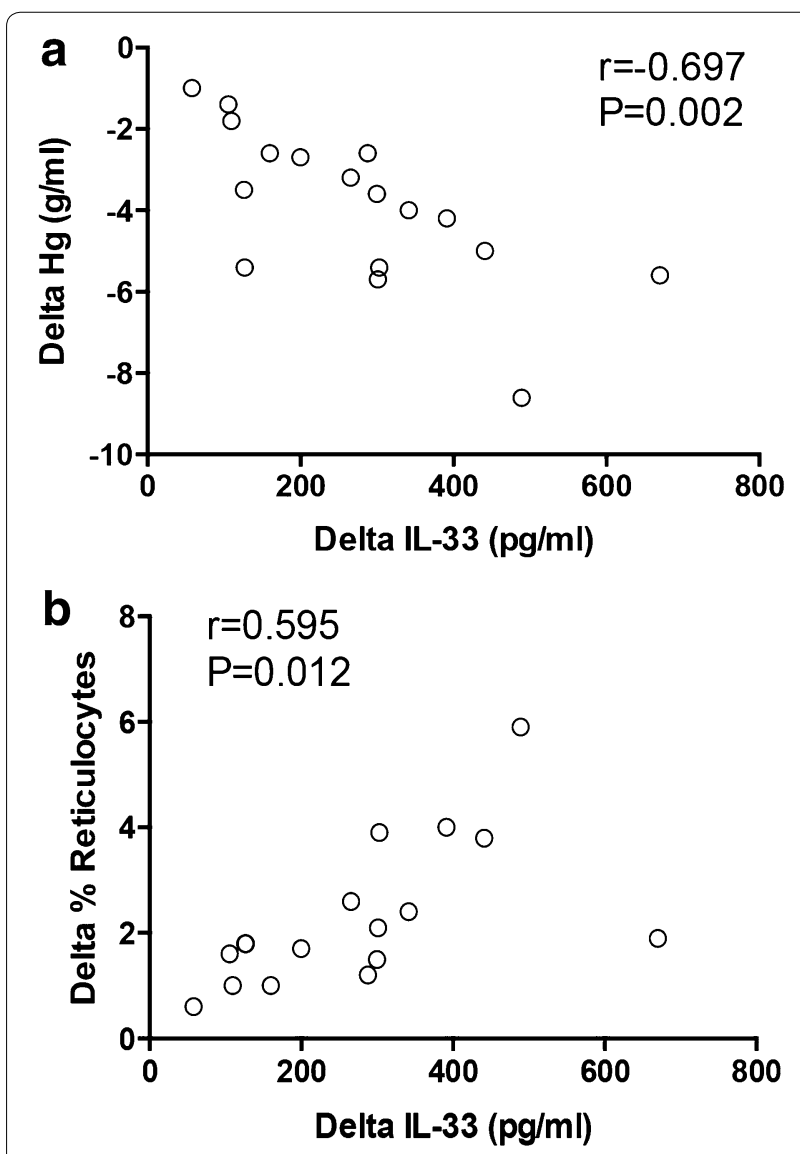

C

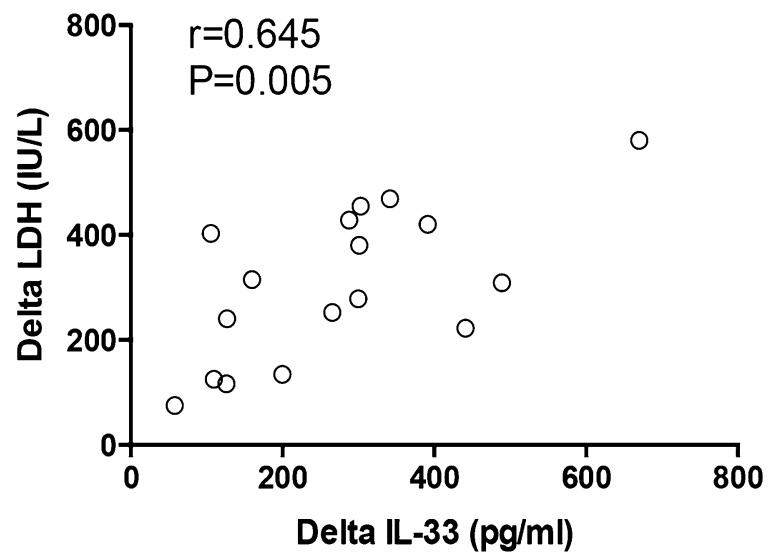

Fig. 2 IL-33 was sensitive to AlHA disease activity. Patients with active AlHA $(n=17)$ were followed up and analyzed for the changes of serum IL-33, reticulocyte count, hemoglobin and LDH. Delta represented the different values between post-follow and pre-follow. The correlation between changes of serum IL-33 and changes of hemoglobin (a), changes of reticulocyte count (b), and changes of $\mathrm{LDH}(\mathbf{c})$ was detected. One dot represented the result from one patient

Currently, therapeutic options for AIHA are usually corticosteroids, splenectomy, intravenous immunoglobulins, plasma-exchange and other immunosuppressive drugs,
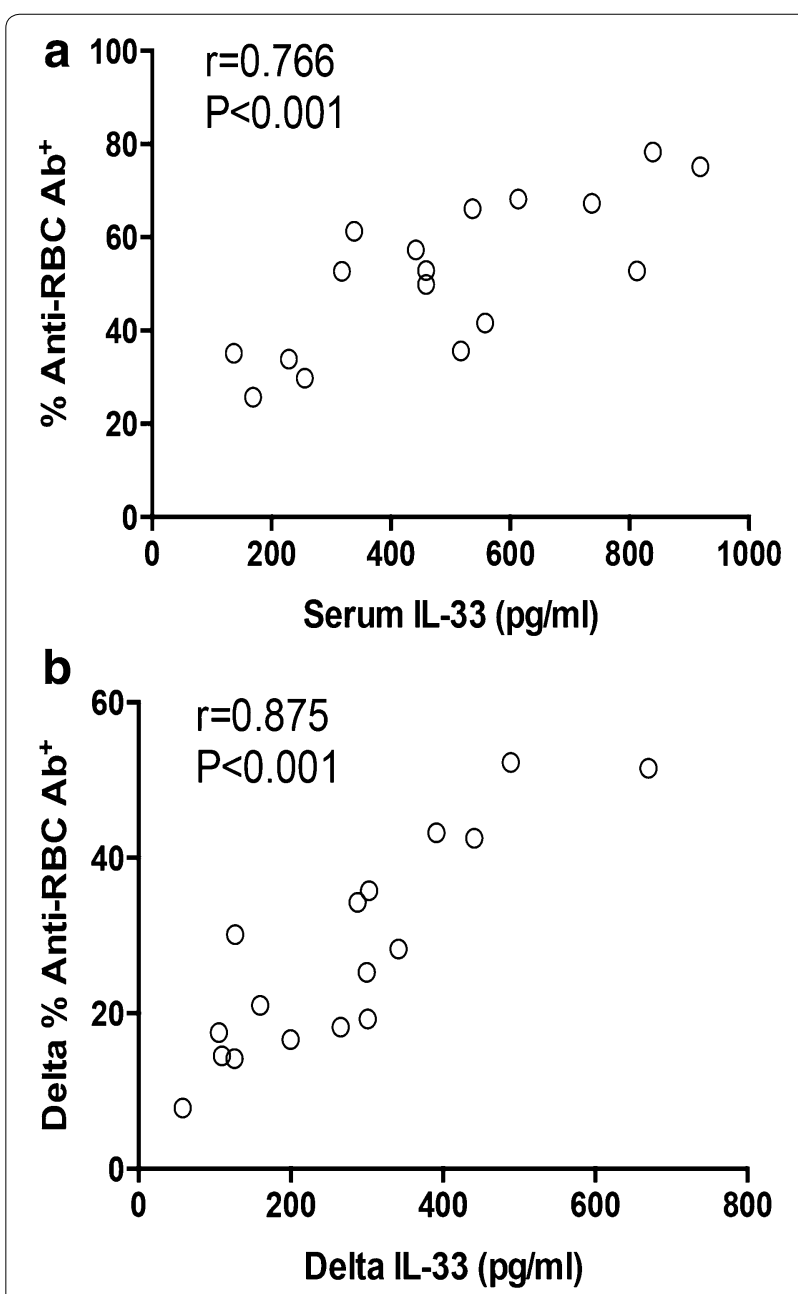

Fig. 3 IL-33 was associated with anti-RBC antibody production. a The correlation of serum IL-33 with anti-RBC antibody level was determined in AlHA patients ( $n=17$ ); $\mathbf{b}$ the correlation between changes of serum IL-33 and changes of anti-RBC antibody production was analyzed in AlHA patients $(n=17)$. One dot represented the result from one patient

which are non-specific and have considerable side effects [2, 22-24]. Rituximab, a monoclonal antibody against the protein $\mathrm{CD} 20$ and can destroy immune system $\mathrm{B}$ cells, has been tested in treatment of AIHA [3, 25-27]. However, autoantibody production in AIHA, especially IgG autoantibody that accounts for warm AIHA development, requires involvement of $\mathrm{T}$ helper cells for B cell responses. Thus, modulation of $\mathrm{T}$ cell responses could be an optimal strategy for AIHA treatment.

IL-33 is a newly described cytokine of IL-1 superfamily and is reported to be involved in $\mathrm{T}$ cell mediated immune responses [15]. Herein, we reported that serum IL-33 was positively associated with and sensitive to anti-RBC autoantibody levels in AIHA patients. 

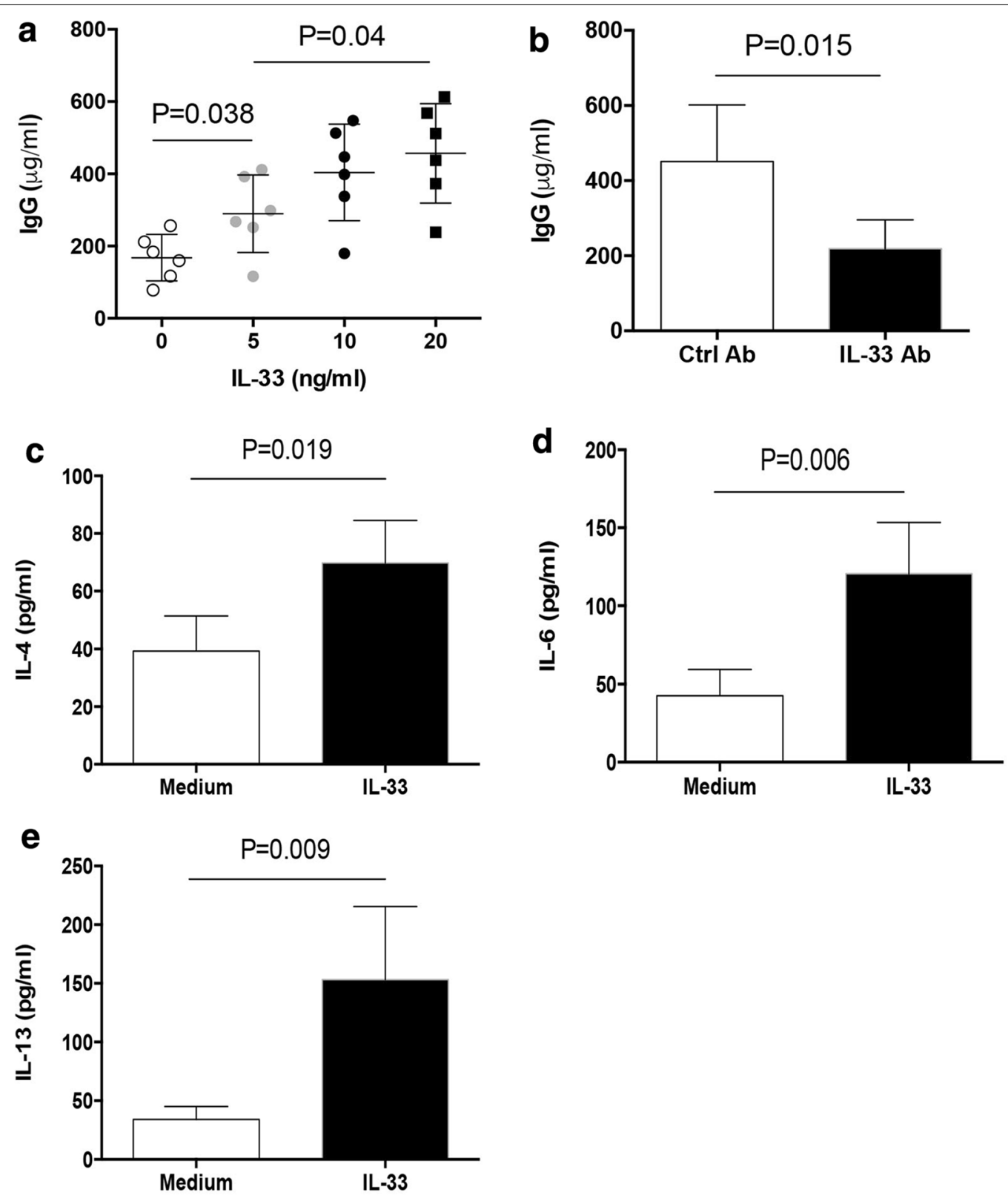

Fig. 4 IL-33 promoted anti-RBC antibody production. a PBMCs from AlHA patients $(n=6)$ were stimulated with anti-lgM, CD4OL in the presence of indicated dose of $\mathrm{IL}-33$ for $6 \mathrm{~d}$, and then assayed for $\mathrm{lg} G$ antibody production. One dot represented the result from one patient; $\mathbf{b}$ PBMCs from AlHA patients $(n=5)$ were stimulated with anti-lgM, CD40L plus IL-33 $(20 \mathrm{ng} / \mathrm{ml})$ in the presence of IL-33 neutralizing antibody or control antibody $(2 \mu \mathrm{mg} /$ $\mathrm{ml}$ ) for $6 \mathrm{~d}$, and detected for lgG antibody; c-e PBMCs from AlHA patients $(\mathrm{n}=4)$ were stimulated with IL-33 $(20 \mathrm{ng} / \mathrm{ml})$ for $72 \mathrm{~h}$, followed by detection for production of IL-4, IL-6 and IL-13 in supernatants

In contrast, serum levels of sST2 in AIHA patients were generally comparable with that in healthy individuals (Additional file 1: Fig. S2), conferring an active function of serum IL-33 in AIHA patients. Further, IL-33 could promote the release of Th2 cytokines and the production of IgG anti-RBC autoantibody. Thus, accumulation of IL-33 in peripheral blood could facilitate the
anti-RBC autoantibody production, which provided a new mechanism through which the autoantibody production was exacerbated in AIHA patients. Therefore, it is reasonable to speculate that IL-33 might be a promising target for controlling autoantibody production and thus restrains AIHA development. In support, we demonstrated that IL-33 could promote the development of 

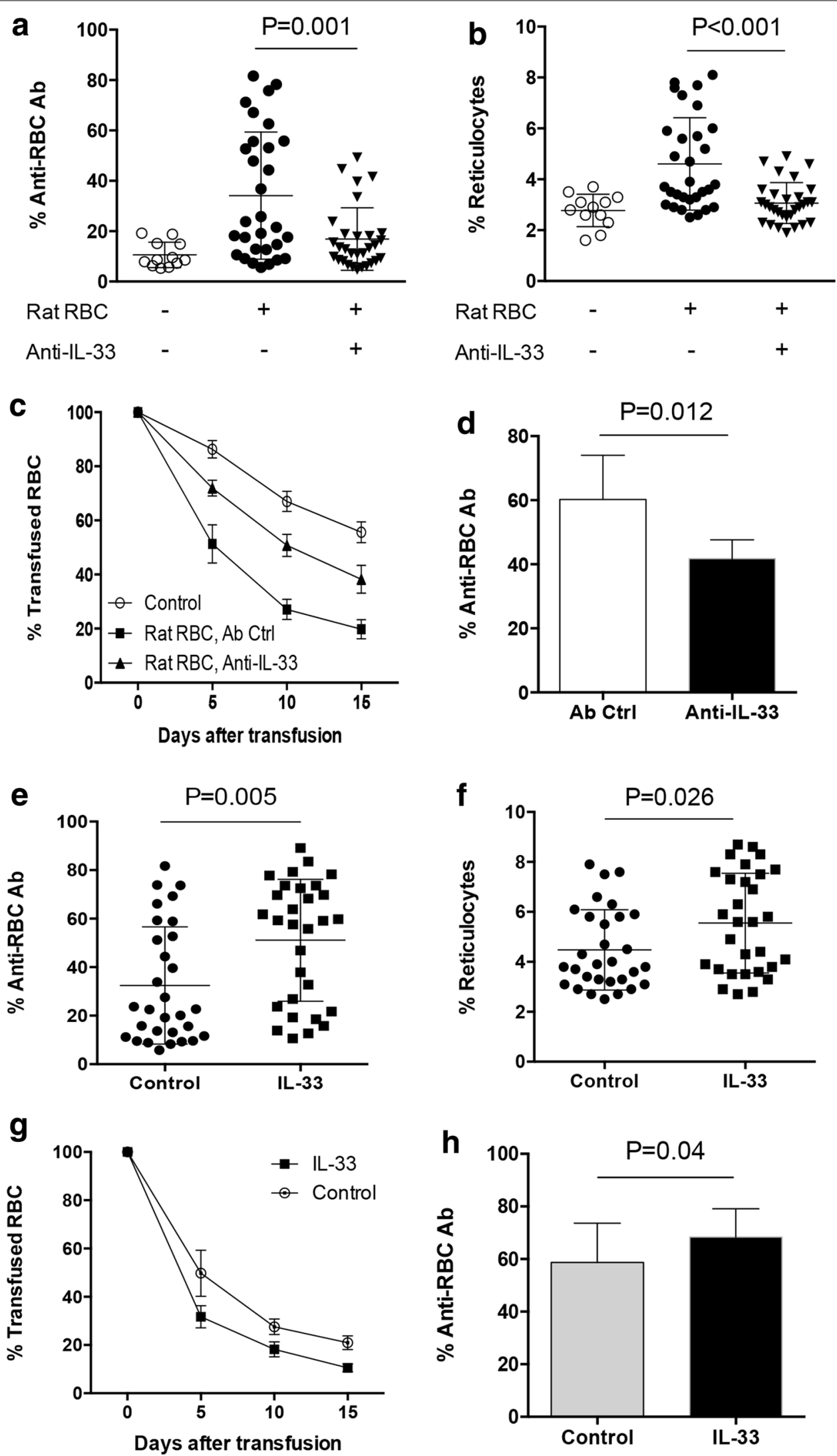
(See figure on previous page.)

Fig. $5 \mathrm{IL}-33$ neutralization restrained AlHA development. a-c Female B6 mice $(n=30)$ were injected with neutralizing antibody to IL-33 or the isotype control, and immunized with rat RBCs for 10 weeks. Then the level of anti-RBC lgG antibody, frequency of circulating reticulocytes and the clearance kinetics of transfused syngeneic mouse RBCs were determined. Each dot represented the results from one mouse. Each bar represented the collective data from control mice $(n=7)$, IL-33 treated AlHA mice $(n=5)$ and Ab Ctrl group $(n=11)$. d The level of anti-RBC IgG antibody in AlHA mice co-injected of IL-33 neutralizing antibody $(n=5)$ or isotype control $(n=13)$. Each dot represented the results from one AlHA mouse. e- $\mathbf{g}$ Female B6 mice ( $n=30$ ) were immunized with rat RBCs plus IL-33 for 10 weeks, and detected for the level of anti-RBC IgG antibody, frequency of circulating reticulocytes and the clearance kinetics of transfused syngeneic mouse RBCs. Each dot represented the results from one mouse. Each bar represented the collective data from five mice. $\mathbf{h}$ Serum level of IgG anti-RBC antibody in AlHA mice with $(n=19)$ or without $(n=12)$ recombinant IL-33 injection. Each bar represented the collective data (mean \pm SD) from each group

AIHA, and more importantly, blockade of IL-33 effectively reduced the incidence of AIHA and ameliorated AIHA disease severity. These results extended previous studies that implicated IL-33 based therapeutics in treatment of a range of diseases including autoimmune disease $[9,15]$.

Herein, we revealed that serum IL-33 was significantly elevated in patients with AIHA and closely correlated with the disease activity. Our results were consistent with previous studies that showed elevated serum IL-33 in autoimmune diseases [28-30]. The increased levels of IL-33 in AIHA patients might result from destructive RBCs and macrophages because RBCs could store and release IL-33, and macrophages served as important scavengers [31, 32]. Our findings suggested that serum level of IL-33 might serve as a potential biomarker for AIHA disease activity. Besides, serum IL-33 was sensitive to changes of disease activity and anti-RBC autoantibody production in AIHA patients, indicating that serum IL-33 was also a promising indicator for monitoring the efficiency of clinical practice. To our knowledge, this is the first study that described the elevated serum IL-33 and its close correlation with disease activity in AIHA patients. Considering the facts that around $5 \%$ of AIHA remains DAT-negative and treatment of AIHA is currently not evidence-based [33], new biomarkers that have diagnostic values or that can be used to assess treatment response could be helpful in clinical practice.

In this study, we observed an interesting effect of IL-33 on anti-RBC antibody production, which might due to increased secretion of Th2 cytokines. In fact, we found diminished levels of Th2 cytokines in AIHA mice coinjected with anti-IL-33 neutralizing antibody, and elevated levels Th2 cytokines in AIHA mice co-injected with IL-33 (Additional file 1: Fig. S3A, B). These Th2 cytokines are considered important for supporting $B$ cell responses. We observed elevated frequency of B cells within PBMCs after stimulation with IL-33 in vitro (Additional file 1: Fig. S4A), and defective development of germinal center B cells in AIHA mice co-injected with anti-IL-33 neutralizing antibody (Additional file 1: Fig. S4B). Further, disrupt IL-6 signaling with neutralizing antibody significantly abrogated the effect of IL-33 on IgG anti-RBC antibody production (Additional file 1: Fig. S5). These findings suggested important roles of IL-33-mediated Th2 cytokines in AIHA pathogenesis. It is well acknowledged that ST2 is highly expressed on group 2 innate lymphoid cells and $\mathrm{T}$ helper 2 cells [34]. Therefore, we proposed that production of anti-RBC antibody in AIHA patients was, at least in part, dependent on $\mathrm{ST}_{2}{ }^{+} \mathrm{T}$ cells, which released increased levels of Th2 cytokines in response to IL-33 and thus support $\mathrm{B}$ cell responses. The destructive RBCs and engaged macrophages would promote the accumulation of IL-33 and feed-forward the generation of anti$\mathrm{RBC}$ antibody in AIHA patients.

\section{Conclusions}

Here we reported an elevated serum IL-33 and its contribution to anti-RBC autoantibody production in patients with AIHA. IL-33 was a potential biomarker for monitoring disease activity and treatment response in AIHA patients. Targeting IL-33 was a promising therapeutic strategy for AIHA patients in clinical practice.

\section{Additional file}

Additional file 1: Figures. Gating strategy for anti-RBC antibody analysis, serum levels of sST2 in AlHA patients, Th2 cytokines in AlHA mice coinjected with IL-33 protein or IL-33 neutralizing antibody, B cell response to IL-33 stimulation or IL-33 blockade, and effect of IL-6 on IL-33-mediated autoantibody production were presented.

\section{Abbreviations}

AlHA: autoimmune hemolytic anemia; RBC: red blood cell; IL-33: interleukin 33; PBMC: peripheral blood mononuclear cells; LDH: lactate dehydrogenase.

\section{Authors' contributions}

XB carried out experiments, participated in study design and data analysis. TZ provided important regents and performed experiments. CW participated in study design and performed data analysis. TR participated in study design and coordination and helped to perform experiments. ZW conceived of the study and wrote the manuscript. All authors read and approved the final manuscript.

\footnotetext{
Author details

${ }^{1}$ Department of Clinical Laboratory, Qingdao Women \& Children Hospital, Qingdao 266034, Shandong, China. ${ }^{2}$ Department of Oncology, Qingdao Municipal Hospital, Qingdao 266071, Shandong, China. ${ }^{3}$ Department of Respiratory Medicine, East Hospital, Tongji University School of Medicine, 150
} 
Jimo Road, Shanghai 200120, China. ${ }^{4}$ Institute of Immunobiology, Shanghai Medical College of Fudan University, Shanghai 200032, China. ${ }^{5}$ Present Address: Division of Immunology and Rheumatology, Stanford University School of Medicine, 269 Campus Drive West, Stanford, CA 94305, USA.

\section{Acknowledgements}

This work was supported in part by Qingdao Municipal Science and Technology Commission (2010KZJ-9), Qingdao Public Health Bureau (2012-WSZD042), National Natural Science Foundation of China $(81372347,81402555)$ and Key Disciplines in Pudong New Area of Shanghai City (PWZx2014-10). These funding provided financial support for conduct of the research without any involvement in study design, data analysis and manuscript preparation.

\section{Competing interests}

The authors declare that they have no competing interests.

Received: 15 June 2015 Accepted: 30 November 2015

Published online: 16 December 2015

\section{References}

1. Xu L, Zhang T, Liu Z, Li Q, Xu Z, Ren T. Critical role of Th17 cells in development of autoimmune hemolytic anemia. Exp Hematol. 2012;40:994-1004.

2. Zanella A, Barcellini W. Treatment of autoimmune hemolytic anemias. Haematologica. 2014;99:1547-54.

3. Stasi R. Rituximab in autoimmune hematologic diseases: not just a matter of B cells. Semin Hematol. 2010;47:170-9.

4. Mqadmi A, Zheng X, Yazdanbakhsh K. CD4+ CD25+ regulatory T cells control induction of autoimmune hemolytic anemia. Blood. 2005;105:3746-8.

5. Michel M. Classification and therapeutic approaches in autoimmune hemolytic anemia: an update. Expert Rev Hematol. 2011;4:607-18.

6. Berentsen S, Sundic T. Red blood cell destruction in autoimmune hemolytic anemia: role of complement and potential new targets for therapy. Biomed Res Int. 2015;2015:363278.

7. Pastorelli L, De Salvo C, Vecchi M, Pizarro TT. The role of IL-33 in gut mucosal inflammation. Mediators Inflamm. 2013;2013:608187.

8. Cayrol C, Girard JP. IL-33: an alarmin cytokine with crucial roles in innate immunity, inflammation and allergy. Curr Opin Immunol. 2014;31:31-7.

9. Kakkar R, Lee RT. The IL-33/ST2 pathway: therapeutic target and novel biomarker. Nat Rev Drug Discov. 2008;7:827-40.

10. Shen J, Shang Q, Wong CK, Li EK, Wang S, Li RJ, et al. IL-33 and soluble ST2 levels as novel predictors for remission and progression of carotid plaque in early rheumatoid arthritis: a prospective study. Semin Arthritis Rheum. 2015. doi:10.1016/j.semarthrit.2015.02.001.

11. Duan L, Chen J, Gong F, Shi G. The role of IL-33 in rheumatic diseases. Clin Dev Immunol. 2013;2013:924363.

12. Hong YS, Moon SJ, Joo YB, Jeon CH, Cho ML, Ju JH, et al. Measurement of interleukin-33 (IL-33) and IL-33 receptors (SST2 and ST2L) in patients with rheumatoid arthritis. J Korean Med Sci. 2011;26:1132-9.

13. Matsuyama Y, Okazaki H, Hoshino M, Onishi S, Kamata Y, Nagatani K, et al. Sustained elevation of interleukin-33 in sera and synovial fluids from patients with rheumatoid arthritis non-responsive to anti-tumor necrosis factor: possible association with persistent IL-1 $\beta$ signaling and a poor clinical response. Rheumatol Int. 2012;32:1397-401.

14. Xu WD, Zhang M, Zhang YJ, Ye DQ. IL-33 in rheumatoid arthritis: potential role in pathogenesis and therapy. Hum Immunol. 2013;74:1057-60.

15. Wang S, Ding L, Liu SS, Wang C, Leng RX, Chen GM, et al. IL-33: a potential therapeutic target in autoimmune diseases. J Investig Med. 2012:60:1151-6.

16. Yang Z, Liang Y, Xi W, Li C, Zhong R. Association of increased serum IL-33 levels with clinical and laboratory characteristics of systemic lupus erythematosus in Chinese population. Clin Exp Med. 2011;11:75-80.

17. Yu SL, Wong CK, Tam LS. The alarmin functions of high-mobility group box-1 and IL-33 in the pathogenesis of systemic lupus erythematosus. Expert Rev Clin Immunol. 2013;9:739-49.

18. Li $P$, Lin W, Zheng X. IL-33 neutralization suppresses lupus disease in lupus-prone mice. Inflammation. 2014;37:824-32.
19. Hoyer KK, Kuswanto WF, Gallo E, Abbas AK. Distinct roles of helper T-cell subsets in a systemic autoimmune disease. Blood. 2009;113:389-95.

20. Chaudhary RK, Das SS. Autoimmune hemolytic anemia: from lab to bedside. Asian J Transfus Sci. 2014:8:5-12.

21. Hosoba S, Jaye DL, Cohen C, Roback JD, Waller EK. Successful treatment of severe immune hemolytic anemia after allogeneic stem cell transplantation with bortezomib: report of a case and review of literature. Transfusion. 2015;55:259-64.

22. Barros MM, Blajchman MA, Bordin JO. Warm autoimmune hemolytic anemia: recent progress in understanding the immunobiology and the treatment. Transfus Med Rev. 2010;24:195-210.

23. Li BJ, Yuan X, Jiang YJ, Ning-Li, Shu XW, Liu KL. Retrospective analysis of 30 severe autoimmune hemolytic anemia patients treated by whole blood exchange transfusion. Transfusion. 2015. doi:10.1111/trf.13122.

24. Ruivard M, Tournilhac O, Montel S, Fouilhoux AC, Quainon F, Lénat A, et al. Plasma exchanges do not increase red blood cell transfusion efficiency in severe autoimmune hemolytic anemia: a retrospective case-control study. J Clin Apher. 2006;21:202-6.

25. Reynaud Q, Durieu I, Dutertre M, Ledochowski S, Durupt S, Michallet AS, et al. Efficacy and safety of rituximab in auto-immune hemolytic anemia: a meta-analysis of 21 studies. Autoimmun Rev. 2015;14:304-13.

26. So MW, Koo BS, Kim YJ, Kim YG, Lee CK, Yoo B. Successful rituximab treatment of refractory hemophagocytic lymphohistiocytosis and autoimmune hemolytic anemia associated with systemic lupus erythematosus. Mod Rheumatol. 2014;24:855-7.

27. Radhi M, Rumelhart S, Tatman D, Goldman F. Severe autoimmune hemolytic anemia after unrelated umbilical cord blood transplant for familia hemophagocytic lymphohistiocytosis: significant improvement after treatment with rituximab. J Pediatr Hematol Oncol. 2007:29:125-7.

28. Tirado-Sánchez A, Bonifaz A, Ponce-Olivera RM. Elevated IL-33 and SST2 levels in sera of patients with pemphigus vulgaris. Correlation with clinical and immunological parameters during follow up. Br J Dermatol. 2015. doi:10.1111/bjd.13716.

29. Jafarzadeh A, Mohammadi-Kordkhayli M, Ahangar-Parvin R, Azizi V, Khoramdel-Azad H, Shamsizadeh A, et al. Ginger extracts influence the expression of IL-27 and IL-33 in the central nervous system in experimental autoimmune encephalomyelitis and ameliorates the clinical symptoms of disease. J Neuroimmunol. 2014;276:80-8.

30. Fuschiotti P, Larregina AT, Ho J, Feghali-Bostwick C, Medsger TA Jr. Interleukin-13-producing CD8+T cells mediate dermal fibrosis in patients with systemic sclerosis. Arthritis Rheum. 2013;65:236-46.

31. Wei J, Zhao J, Schrott V, Zhang Y, Gladwin M, Bullock G, et al. Red Blood Cells Store and Release Interleukin-33. J Investig Med. 2015;63:806-10.

32. Gunawardena D, Gamakaranage GA. A case of warm autoimmune haemolytic anaemia with intravascular haemolysis: a rare presentation. Ceylon Med J. 2013;58:176-8.

33. Barcellini W. Immune hemolysis: diagnosis and treatment recommendations. Semin Hematol. 2015:52:304-12.

34. Molofsky AB, Savage AK, Locksley RM. Interleukin-33 in tissue homeostasis, injury, and inflammation. Immunity. 2015;42:1005-19.

\section{Submit your next manuscript to BioMed Central and we will help you at every step:}

- We accept pre-submission inquiries

- Our selector tool helps you to find the most relevant journal

- We provide round the clock customer support

- Convenient online submission

- Thorough peer review

- Inclusion in PubMed and all major indexing services

- Maximum visibility for your research

Submit your manuscript at www.biomedcentral.com/submit
C Biomed Central 